\title{
Tensor Product of 2-Frames in 2-Hilbert Spaces
}

\author{
G. Upender Reddy \\ Department of Mathematics, University College of Science \& Informatics, Mahatma Gandhi University, \\ Nalgonda, India \\ Email: upendermathsmgu@gmail.com
}

Received 9 October 2015; accepted 2 July 2016; published 5 July 2016

Copyright (C) 2016 by author and Scientific Research Publishing Inc.

This work is licensed under the Creative Commons Attribution International License (CC BY).

http://creativecommons.org/licenses/by/4.0/

c) (7) Open Access

\begin{abstract}
2-frames in 2-Hilbert spaces are studied and some results on it are presented. The tensor product of 2-frames in 2-Hilbert spaces is introduced. It is shown that the tensor product of two 2 -frames is a 2-frame for the tensor product of Hilbert spaces. Some results on tensor product of 2-frames are established.
\end{abstract}

Keywords

Tensor Product, 2-Inner Product Spaces, Frames, 2-Frames

\section{Introduction}

The concept of frames in Hilbert spaces has been introduced by Duffin and Schaefer in 1952 to study some deep problems in nonharmonic Fourier series. D. Han and D.R. Larson [1] have developed a number of basic aspects of operator-theoretic approach to frame theory in Hilbert space. Peter G. Casazza [2] presented a tutorial on frame theory and he suggested the major directions of research in frame theory.

The concept of linear 2-normed spaces has been investigated by S. Gahler in 1965 [3] and has been developed extensively in different subjects by many authors. A concept which is related to a 2-normed space is 2-inner product space which has been intensively studied by many mathematicians in the last three decades. The concept of 2-frames for 2-inner product spaces was introduced by Ali Akbar Arefijammaal and Ghadir Sadeghi [4] and described some fundamental properties of them. Y. J. Cho, S. S. Dragomir, A. White and S. S. Kim [5] are presented some inequalities in 2-inner product spaces. Some results on 2-inner product spaces are described by H. Mazaherl and R. Kazemi [6]. The tensor product of frames in tensor product of Hilbert spaces is introduced by G. Upender Reddy and N. Gopal Reddy [7] and some results on tensor frame operator are presented. 
In this paper, 2-frames in 2-Hilbert spaces are studied and some results on it are presented. The tensor product of 2-frames in 2-Hilbert spaces is introduced. It is shown that the tensor product of two 2-frames is a 2-frame for the tensor product of Hilbert spaces. Some results on tensor product of 2-frames are established.

\section{Preliminaries}

The following definitions from [2] [5] are useful in our discussion.

Definition 2.1. A sequence $\left\{x_{i}\right\}_{i=1}^{\infty}$ of vectors in a Hilbert space $X$ is called a frame if there exist constants 0 $<\mathrm{A} \leq \mathrm{B}<\propto$ such that

$$
A\|x\|^{2} \leq \sum_{i=1}^{\infty}\left|\left\langle x, x_{i}\right\rangle\right|^{2} \leq B\|x\|^{2} \text { for all } x \in X .
$$

The above inequality is called the frame inequality. The numbers $A$ and $B$ are called lower and upper frame bounds respectively.

Definition 2.2. A synthesis operator $T: l_{2} \rightarrow X$ is defined as $T e_{i}=x_{i}$ where $\left\{e_{i}\right\}$ is an orthonormal basis for $l_{2}$.

Definition 2.3. Let $\left\{x_{i}\right\}_{i=1}^{\infty}$ be a frame for $X$ and $\left\{e_{i}\right\}$ be an orthonormal basis for $l_{2}$. Then, the analysis operator $T^{*}: X \rightarrow l_{2}$ is the adjoint of synthesis operator $T$ and is defined as $T^{*} x=\sum_{i=1}^{\infty}\left\langle x, x_{i}\right\rangle e_{i}$ for all $x \in X$.

Definition 2.4. Let $\left\{x_{i}\right\}_{i=1}^{\infty}$ be a frame for the Hilbert space $H$. A frame operator $S=T T^{*}: X \rightarrow X$ is defined as $S x=\sum_{i=1}^{\infty}\left\langle x, x_{i}\right\rangle x_{i}$ for all $x \in X$.

Here we give the basic definitions of 2-normed spaces and 2-inner product spaces from [3] [6].

Definition 2.5. $X$ be a real linear space of dimension greater than 1 and let $\|.,$.$\| be a real-valued function on$ $X \times X$ satisfying the following conditions.
a) $\|x, y\| \geq 0$ and $\|x, y\|=0$ if and only if $x$ and $y$ are linearly dependent vectors.
b) $\|x, y\|=\|y, x\|$ for all $x, y \in X$.
c) $\|\alpha x, y\|=|\alpha|\|x, y\|$ for any real number $\alpha$ and for all $x, y \in X$.
d) $\|x+y, z\| \leq\|x, z\|+\|y, z\|$ for all $x, y, z \in X$.

Then $\|.,$.$\| is called 2-norm on X$ and $(X,\|.,\|$.$) called a linear 2-normed space.$

Definition 2.6. Let $X$ be a linear space of dimension greater than 1 over the field $K(=R$ or $C$ ). Suppose that (.,./.) is $K$-valued function on $X \times X \times X$ which satisfies the following conditions.
b) $(x, x / z)=(z, z / x)$.
c) $(y, x / z)=\overline{(x, y / z)}$.
d) $(\alpha x, y / z)=\alpha(x, y / z)$ for all $\alpha \in K$.
e) $\left(x_{1}+x_{2}, y / z\right)=\left(x_{1}, y / z\right)+\left(x_{2}, y / z\right)$.

a) $(x, x / z) \geq 0$ and $(x, x / z)=0$ if and only if $x$ and $z$ are linearly dependent.

Then $(., . /$.$) is called a 2-inner product on X$ and $(X,(., . /)$.$) is called a 2-inner product space (or 2-pre$ Hilbert space).

If $(X,\langle\rangle)$ is an inner product space, then the standard 2-inner product space $(., . /$.$) is defined on X$ by

$$
(x, y / z)=\left|\begin{array}{ll}
\langle x, y\rangle & \langle x, z\rangle \\
\langle z, y\rangle & \langle z, z\rangle
\end{array}\right|=\langle x, y\rangle\langle z, z\rangle-\langle x, z\rangle\langle z, y\rangle \text { for all } x, y, z \in X .
$$

Let $(X,(., . /)$.$) be a 2-inner product space, we can define a 2-norm on X \times X$ by $\|x, y\|=(x, x / y)^{\frac{1}{2}}$, for all $x, y \in X$.

Using the above properties, we can prove the Cauchy-Schwartz inequality $(x, y / b)^{2} \leq\|x, b\|^{2}\|y, b\|^{2}$.

A 2-inner product space $X$ is called a 2-Hilbert space if it is complete. 


\section{2-Frames}

The definition of 2-frame from [1] as follows.

Definition 3.1 Let $(X,(., /)$.$) be a 2-Hilbert space and \xi \in X$. A sequence $\left\{x_{i}\right\}_{i=1}^{\infty}$ of elements in $X$ is called a 2-frame associated to $\xi$ if there exist $0<A \leq B<\propto$ such that

$$
A\|x, \xi\|^{2} \leq \sum_{i=1}^{\infty}\left|\left(x, x_{i} / \xi\right)\right|^{2} \leq B\|x, \xi\|^{2} \text { for all } x \in X .
$$

The above inequality is called the 2-frame inequality. The numbers $A$ and $B$ are called the lower and upper 2 -frame bounds respectively.

The following proposition [1] shows that in the standard 2-inner product spaces every frame is a 2-frame.

Proposition 3.2. Let $(X,\langle\rangle)$ be a Hilbert space and $\left\{x_{i}\right\}_{i=1}^{\infty}$ is a frame for $H$. Then, it is a 2-frame with the standard 2-inner product space on $X$.

Proof: Suppose that $\left\{x_{i}\right\}_{i=1}^{\infty}$ is a frame for $X$ with frame bounds $A$ and $B$.

$$
\begin{aligned}
\sum_{i=1}^{\infty}\left|\left(x, x_{i} / \xi\right)\right|^{2} & =\sum_{i=1}^{\infty}\left|\left\langle x, x_{i}\right\rangle\langle\xi, \xi\rangle-\langle x, \xi\rangle\left\langle\xi, x_{i}\right\rangle\right|^{2}=\sum_{i=1}^{\infty}\left|\left\langle x, x_{i}\right\rangle-\langle x, \xi\rangle\left\langle\xi, x_{i}\right\rangle\right|^{2} \\
& =\sum_{i=1}^{\infty}\left|\left\langle x-\langle x, \xi\rangle \xi, x_{i}\right\rangle\right|^{2} \leq B\|x-\langle x, \xi\rangle \xi\|^{2}=B\left(\|x\|^{2}-|\langle x, \xi\rangle|^{2}\right) \\
& =B(x, x / \xi)=B\|x, \xi\|^{2} .
\end{aligned}
$$

Then

Similarly we can prove that $A\|x, \xi\|^{2} \leq \sum_{i=1}^{\infty}\left|\left(x, x_{i} / \xi\right)\right|^{2}$. Hence $\left\{x_{i}\right\}_{i=1}^{\infty}$ is a 2-frame for 2-Hilbert space.

Suppose $(X,(., . /)$.$) is a 2-Hilbert space and L_{\xi}$ the subspace generated with a fixed element $\xi$ in $X$. Let $M_{\xi}$ be denote the algebraic complement of $L_{\xi}$ in $X$. So we have $L_{\xi} \oplus M_{\xi}=X$. We define the inner product $\langle., .\rangle_{\xi}$ on $X$ as follows $\langle x, z\rangle_{\xi}=\langle x, z / \xi\rangle$.

A sequence $\left\{x_{i}\right\}_{i=1}^{\infty}$ of elements in $X$ is a 2-frame associated to $\xi$ with frame bounds $A$ and $B$, then the definition of 2-frame can be written as $A\|x\|_{\xi}^{2} \leq \sum_{i=1}^{\infty}\left|\left(x, x_{i}\right)_{\xi}\right|^{2} \leq B\|x\|_{\xi}^{2}$, for all $x \in X$.

Definition 3.3. Let $\left\{x_{i}\right\}_{i=1}^{\infty}$ be a 2-frame in $X$. Then, the 2-Synthesis operator $T_{\xi}: l^{2} \rightarrow X_{\xi}$ is defined by $T_{\xi}\left\{c_{i}\right\}=\sum_{i=1}^{\infty} c_{i} x_{i}$.

Definition 3.4. Let $\left\{x_{i}\right\}_{i=1}^{\infty}$ be a 2 -frame in $X$. Then, the 2-Analysis operator $T_{\xi}^{*}: X_{\xi} \rightarrow l^{2}$ is defined by $T_{\xi}^{*}(x)=\left\{\left(x, x_{i} / \xi\right)\right\}_{i=1}^{\infty}$.

Definition 3.5. Let $\left\{x_{i}\right\}_{i=1}^{\infty}$ be a 2-frame associated to $\xi$ with frame bounds $A$ and $B$ in a 2-Hilbert space $X$. A 2-frame operator $S_{\xi}: X_{\xi} \rightarrow X_{\xi}$ is defined by $S_{\xi} x=\sum_{i=1}^{\infty}\left(x, x_{i} / \xi\right) x_{i}$.

Theorem 3.6. Suppose that $\left\{x_{i}\right\}_{i=1}^{\infty}$ is a sequence in 2-Hilbert space $X$, with $x=\sum_{i=1}^{\infty}\left(x, x_{i} / \xi\right) x_{i}$ holds for all $x \in X$ if and only if $\left\{x_{i}\right\}_{i=1}^{\infty}$ is a 2-normalized tight frame for $X$.

Proof: Since $\left\{x_{i}\right\}_{i=1}^{\infty}$ is a 2-normalized tight frame for $X$, for all $x \in X$

$$
\begin{gathered}
\Leftrightarrow\|x, \xi\|^{2}=\sum_{i=1}^{\infty}\left|\left(x, x_{i} / \xi\right)\right|^{2} \Leftrightarrow\|x, \xi\|^{2}=\sum_{i=1}^{\infty}\left(x, x_{i} / \xi\right)\left(x_{i}, x / \xi\right) \\
\Leftrightarrow(x, x / \xi)=\left(\sum_{i=1}^{\infty}\left(x, x_{i} / \xi\right) x_{i}, x / \xi\right) \Leftrightarrow x=\sum_{i=1}^{\infty}\left(x, x_{i} / \xi\right) x_{i} \text { for all } x \in X .
\end{gathered}
$$


Theorem 3.7. Suppose that $\left\{x_{i}\right\}_{i=1}^{\infty}$ is a 2-frame for Hilbert space $X$, and $T$ is co-isometry. Then $\left\{T x_{i}\right\}_{i=1}^{\infty}$ is a 2-frame for $X$.

Proof: Since $\left\{x_{i}\right\}_{i=1}^{\infty}$ is a 2-frame for $X$, by Definition 3.1, we have

$$
A\|x, \xi\|^{2} \leq \sum_{i=1}^{\infty}\left|\left(x, x_{i} / \xi\right)\right|^{2} \leq B\|x, \xi\|^{2},(x \in X)
$$

Since $T^{*}: X \rightarrow X$ is an operator, for all $x \in H$, we have $T^{*} x \in X$

Therefore, the above Equation (1) is true for $T^{*} x \in X$

$$
\begin{gathered}
A\left\|T^{*} x, \xi\right\|^{2} \leq \sum_{i=1}^{\infty}\left|\left(T^{*} x, x_{i} / \xi\right)\right|^{2} \leq B\left\|T^{*} x, \xi\right\|^{2} \\
A\left\|T^{*} x, \xi\right\|^{2} \leq \sum_{i=1}^{\infty}\left|\left(x, T x_{i} / \xi\right)\right|^{2} \leq B\left\|T^{*} x, \xi\right\|^{2} \text {, for all } x \in X
\end{gathered}
$$

By using the fact that $\mathrm{T}$ is co-isometry, we have

$$
A\|x, \xi\|^{2} \leq \sum_{i=1}^{\infty}\left|\left(x, T x_{i} / \xi\right)\right|^{2} \leq B\|x, \xi\|^{2} \text {, for all } x \in X
$$

Which shows that $\left\{T x_{i}\right\}_{i=1}^{\infty}$ is a 2-frame for $X$.

\section{Tensor Product of 2-Frames}

Let $H_{1}$ and $H_{2}$ be 2-Hilbert spaces with inner products $(., . / .)_{1},(., . /)_{2}$ respectively. The tensor product of $H_{1}$ and $H_{2}$ is denoted by $H_{1} \otimes H_{2}$ and is an inner product space with respect to the inner product given by

$$
\left(x_{1} \otimes x_{2}, y_{1} \otimes y_{2} / z_{1} \otimes z_{2}\right)=\left(x_{1}, y_{1} / z_{1}\right)_{1}\left(x_{2}, y_{2} / z_{2}\right)_{2}
$$

for all $x_{1}, y_{1}, z_{1} \in H_{1}$ and $x_{2}, y_{2}, z_{2} \in H_{2}$. The norm on $H_{1} \otimes H_{2}$ is defined by

$$
\left\|x_{1} \otimes x_{2}, y_{1} \otimes y_{2}\right\|=\left\|x_{1}, y_{1}\right\|_{1}\left\|x_{2}, y_{2}\right\|_{2} \quad \forall x_{1}, y_{1} \in H_{1} \text { and } x_{2}, y_{2} \in H_{2}
$$

where $\|., .\|_{1}$, and $\|., .\|_{2}$ are norms generated by $(., . / .)_{1}$ and $(., . / .)_{2}$ respectively. The space $H_{1} \otimes H_{2}$ is completion with the above inner product. Therefore, the space $H_{1} \otimes H_{2}$ is a 2-Hilbert space.

The following definition is the extension of (3.1) to the sequence $\left\{x_{i} \otimes y_{j}\right\}$.

Definition 4.1. Let $\left\{x_{i}\right\}$ and $\left\{y_{j}\right\}$ be the sequences of vectors in 2-Hilbert spaces $H_{1}$ and $H_{2}$ respectively. Then, the sequence of vectors $\left\{x_{i} \otimes y_{j}\right\}$ is said to be a tensor product of 2 -frame for the tensor product of Hilbert spaces $H_{1} \otimes H_{2}$ associated to $\xi \otimes \eta$ if there exist two constants $0<A \leq B<\propto$ such that

$$
\begin{aligned}
& A\|x \otimes y, \xi \otimes \eta\|^{2} \leq \sum_{i, j}\left|\left(x \otimes y, x_{i} \otimes y_{j} / \xi \otimes \eta\right)\right|^{2} \leq B\|x \otimes y, \xi \otimes \eta\|^{2}, \\
& \text { for all } x \otimes y \in H_{1} \otimes H_{2}
\end{aligned}
$$

The numbers $A$ and $B$ are called lower and upper frame bounds of the tensor product of 2-frame, respectively.

Theorem 4.2. Let $\left\{x_{i}\right\}$ and $\left\{y_{j}\right\}$ be two sequences in Hilbert spaces $H_{1}$ and $H_{2}$ respectively. Then, the sequence $\left\{x_{i} \otimes y_{j}\right\}$ is a tensor product of 2-frame for $H_{1} \otimes H_{2}$ if and only if $\left\{x_{i}\right\}$ and $\left\{y_{j}\right\}$ are the 2-frames for $H_{1}$ and $H_{2}$ respectively.

Proof. Suppose that $\left\{x_{i} \otimes y_{j}\right\}$ is a 2-frame for $H_{1} \otimes H_{2}$ associated to $\xi \otimes \eta$. Then, for each $x \otimes y \in H_{1} \otimes H_{2}-\{0 \otimes 0\}$

$$
\begin{aligned}
& A\|x \otimes y, \xi \otimes \eta\|^{2} \leq \sum_{i, j}\left|\left(x \otimes y, x_{i} \otimes y_{j} / \xi \otimes \eta\right)\right|^{2} \leq B\|x \otimes y, \xi \otimes \eta\|^{2}, \\
& \text { for all } x \otimes y \in H_{1} \otimes H_{2}
\end{aligned}
$$

On using (2) and (3) the above equation becomes 


$$
A(x, x / \xi)_{1}(y, y / \eta)_{2} \leq \sum_{i}\left|\left(x, x_{i} / \xi\right)_{1}\right|^{2} \sum_{j}\left|\left(y, y_{j} / \eta\right)_{2}\right|^{2} \leq B(x, x / \xi)_{1}(y, y / \eta)_{2} .
$$

This gives $\frac{A(y, y / \eta)_{2}}{\sum_{j}\left|\left(y, y_{j} / \eta\right)_{2}\right|^{2}}(x, x / \xi)_{1} \leq \sum_{i}\left|\left(x, x_{i} / \xi\right)_{1}\right|^{2} \leq \frac{B(y, y / \eta)_{2}}{\sum_{j}\left|\left(y, y_{j} / \eta\right)_{2}\right|^{2}}(x, x / \xi)_{1}$.

That is $A_{1}(x, x / \xi)_{1} \leq \sum_{i}\left|\left(x, x_{i} / \xi\right)\right|^{2} \leq B_{1}\left(x, x_{i} / \xi\right)_{1}$, for all $x \in H_{1}$.

Therefore $A_{1}\|x, \xi\|^{2} \leq \sum_{i}\left|\left(x, x_{i} / \xi\right)_{1}\right|^{2} \leq B_{1}\|x, \xi\|^{2}$, for all $x \in H_{1}$,

where $A_{1}=\frac{A(y, y / \eta)_{2}}{\sum_{j}\left|\left(y, y_{j} / \eta\right)_{2}\right|^{2}}$ and $B_{1}=\frac{B(y, y / \eta)_{2}}{\sum_{j}\left|\left(y, y_{j} / \eta\right)_{2}\right|^{2}}$.

Which shows that $\left\{x_{i}\right\}$ is a 2-frame for $H_{1}$ associated to $\xi$. Similarly we can prove that $\left\{y_{j}\right\}$ is a 2frame for $\mathrm{H}_{2}$ associated to $\eta$.

Conversely, assume that $\left\{x_{i}\right\}$ is a 2-frame for $H_{1}$ associated to $\xi$ with frame bounds $A_{1}, B_{1}$ and $\left\{y_{j}\right\}$ is a 2-frame for $H_{2}$ associated to $\eta$ with frame bounds $A_{2}, B_{2}$. Then

$$
A_{1}\|x, \xi\|_{1}^{2} \leq \sum_{i}\left|\left(x, x_{i} / \xi\right)_{1}\right|_{1}^{2} \leq B_{1}\|x, \xi\|_{1}^{2} \text {, for all } x \in H_{1}
$$

and

$$
A_{2}\|y, \eta\|_{2}^{2} \leq \sum_{j}\left|\left(y, y_{i} / \eta\right)_{2}\right|_{2}^{2} \leq B_{2}\|y, \eta\|_{2}^{2} \text {, for all } y \in H_{2}
$$

multiplying the Equations (4) and (5) we get

$$
A_{1} A_{2}\|x \otimes y, \xi \otimes \eta\|^{2} \leq \sum_{i, j}\left|\left(x \otimes y, x_{i} \otimes y_{j} / \xi \otimes \eta\right)\right|^{2} \leq B_{1} B_{2}\|x \otimes y, \xi \otimes \eta\|^{2} \text {, for all } x \otimes y \in H_{1} \otimes H_{2}
$$

Which shows that $\left\{x_{i} \otimes y_{j}\right\}$ is a tensor product of frame for $H_{1} \otimes H_{2}$.

Hence we can have the following remark.

Remark 4.3. If the sequences $\left\{x_{i}\right\},\left\{y_{j}\right\}$ and $\left\{x_{i} \otimes y_{j}\right\}$ are the 2-frames for the Hilbert spaces $H_{1}, H_{2}$ and $H_{1} \otimes H_{2}$ respectively and $S_{\xi}, S_{\eta}$ and $S_{\xi \otimes \eta}$ are the frame operators respectively of above frames, then from 3.5, we have the following.

$$
\begin{gathered}
S_{\xi} x=\sum_{i}\left(x, x_{i} / \xi\right) x_{i}, \quad S_{\eta} x=\sum_{j}\left(y, y_{j} / \eta\right) y_{j} \\
S_{\xi \otimes \eta}(x \otimes y)=\sum_{, i, j}\left(x \otimes y, x_{i} \otimes y_{j} / \xi \otimes \eta\right)\left(x_{i} \otimes y_{j}\right), \quad x \in H_{1}, y \in H_{2}, x \otimes y \in H_{1} \otimes H_{2}
\end{gathered}
$$

Theorem 4.4. If $\left\{x_{i}\right\},\left\{y_{j}\right\}$ and $\left\{x_{i} \otimes y_{j}\right\}$ are the frames for the Hilbert spaces $H_{1}, H_{2}$ and $H_{1} \otimes H_{2}$ with the frame operators $S_{\xi}, S_{\eta}$ and $S_{\xi \otimes \eta}$ respectively, then $S_{\xi \otimes \eta}=S_{\xi} \otimes S_{\eta}$.

Proof. For $x \otimes y \in H_{1} \otimes H_{2}$, we have

$$
\begin{aligned}
S_{\xi \otimes \eta}(x \otimes y) & =\sum_{i, j}\left(x \otimes y, x_{i} \otimes y_{j} / \xi \otimes \eta\right)\left(x_{i} \otimes y_{j}\right) \\
& =\sum_{i, j}\left(x, x_{i} / \xi\right)_{1}\left(y, y_{j} / \eta\right)_{2}\left(x_{i} \otimes y_{j}\right) \\
& =\sum_{i}\left(x, x_{i} / \xi\right)_{1} x_{i} \otimes \sum_{j}\left(y, y_{j} / \eta\right)_{2} y_{j} \\
& =S_{\xi} x \otimes S_{\eta} y=\left(S_{\xi} \otimes S_{\eta}\right)(x \otimes y)
\end{aligned}
$$

Hence $S_{\xi \otimes \eta}=S_{\xi} \otimes S_{\eta}$. 
The following two theorems are the extension of 3.6 and 3.7 to the sequence $\left\{x_{i} \otimes y_{j}\right\}$ so, proofs are left to the reader.

Theorem 4.5. Assume that $\left\{x_{i} \otimes y_{j}\right\}$ is a sequence in a Hilbert space $H_{1} \otimes H_{2}$. Then $x \otimes y=\sum_{i, j}\left(x \otimes y, x_{i} \otimes y_{j} / \xi \otimes \eta\right)\left(x_{i} \otimes y_{j}\right), \quad x \otimes y \in H_{1} \otimes H_{2}$ if and only if $\left\{x_{i} \otimes y_{j}\right\}$ is a 2-normalized tight frame for $H_{1} \otimes H_{2}$.

Theorem 4.6. Suppose that $\left\{x_{i} \otimes y_{j}\right\}$ is a tensor product of 2-frame for $H_{1} \otimes H_{2}$, and $T_{1} \otimes T_{2}$ is co-isometry. Then $\left\{\left(T_{1} \otimes T_{2}\right)\left(x_{i} \otimes y_{j}\right)\right\}$ is a tensor product of 2-frame for $H_{1} \otimes H_{2}$.

\section{Acknowledgements}

The research of the author is partially supported by the UGC (India) [Letter No. F.20-4(1)/2012(BSR)].

\section{References}

[1] Han, D. and Larson, D.R. (2000) Frames, Bases and Group Representations. Memoirs of the AMS.

[2] Casazza, P.G. (2000) The Art of Frame Theory. Taiwanese Journal of Mathematics, 4, 129-201.

[3] Gahler, S. (1965) Lineare 2-Normierte Raume. Mathematische Nachrichten, 28, 1-43. http://dx.doi.org/10.1002/mana.19640280102

[4] Arefijammaal, A.A. and Sadeghi, G. (2013) Frames in 2-Inner Product Spaces. International Journal of Mathematical Sciences and Informatics, 8, 123-130.

[5] Cho, Y.J., Dragomir, S.S., White, A. and Kim, S.S. (1999) Some Inequalities in 2-Inner Product Spaces. Demonstratio Mathematica, 32, 485-493.

[6] Mazaherl, H. and Kazemi, R. (2007) Some Results on 2-Inner Product Spaces. Novi Sad Journal of Mathematics, 37, 35-40.

[7] Reddy, G.U., Reddy, N.G. and Reddy, B.K. (2009) Frame Operator and Hilbert-Schmidt Operator in Tensor Product of Hilbert Spaces. Journal of Dynamical Systems \& Geometric Theories, 7, 61-70.

\section{Submit or recommend next manuscript to SCIRP and we will provide best service for you:}

Accepting pre-submission inquiries through Email, Facebook, Linkedin, Twitter, etc A wide selection of journals (inclusive of 9 subjects, more than 200 journals)

Providing a 24-hour high-quality service

User-friendly online submission system

Fair and swift peer-review system

Efficient typesetting and proofreading procedure

Display of the result of downloads and visits, as well as the number of cited articles

Maximum dissemination of your research work

Submit your manuscript at: http://papersubmission.scirp.org/ 